\title{
Relationship between survival and increased radiation dose to subventricular zone in glioblastoma is controversial
}

\author{
Olgun Elicin · Ebrar Inac • Esengul Kocak Uzel • \\ Songul Karacam • Omer Erol Uzel
}

Received: 25 December 2013/ Accepted: 13 March 2014/Published online: 26 March 2014

(C) Springer Science+Business Media New York 2014

\begin{abstract}
To test the hypothesis on prolonged survival in glioblastoma cases with increased subventricular zone (SVZ) radiation dose. Sixty glioblastoma cases were previously treated with adjuvant radiotherapy and Temozolamide. Ipsilateral, contralateral and bilateral SVZs were contoured and their doses were retrospectively evaluated. Median follow-up, progression free survival (PFS) and overall survival (OS) were 24.5, 8.5 and 19.3 months respectively. Log-rank tests showed a statistically significant correlation between contralateral SVZ (cSVZ) dose $>59.2$ Gy (75th percentile) and poor median PFS (10.37 [95 \% CI 8.37-13.53] vs 7.1 [95\% CI 3.5-8.97] months, $p=0.009)$. cSVZ dose $>59.2$ Gy was associated with poor OS in the subgroup with subtotal resection/biopsy (HR: 4.83 [95 \% CI 1.71-13.97], $p=0.004$ ). High ipsilateral SVZ dose of $>62.25 \mathrm{~Gy}$ ( 75 th percentile) was associated with poor PFS in both subgroups of high performance status (HR: 2.58 [95 \% CI 1.03-6.05], $p=0.044$ ) and SVZ without tumoral contact (HR: 10.57 [95 \% CI 2.04-49], $p=0.008$ ). The effect of high cSVZ dose on PFS lost its statistical significance in multivariate
\end{abstract}

O. Elicin · E. Inac $\cdot$ S. Karacam · O. E. Uzel

Radiation Oncology Department, Cerrahpasa Faculty of Medicine, Istanbul University, I.U. Cerrahpasa Tip Fakultesi, Radyasyon Onkolojisi A.D. - Fatih, 34098 Istanbul, Turkey

\section{O. Elicin $(\bowtie)$}

Inselspital, Radiation Oncology Department, University of Bern, Inselspital Klinik und Poliklinik für Radio-Onkologie,

3010 Bern, Switzerland

e-mail: olgunelicin@gmail.com

\section{E. K. Uzel}

Radiation Oncology Department, Sisli Etfal Teaching Hospital, Sisli Etfal Egitim Arastirma Hastanesi, Radyasyon Onkolojisi Klinigi - Sisli, 34371 Istanbul, Turkey
Cox regression analysis. We report contradictory results compared to previous publications. Changing the clinical practice based on retrospective studies which even do not indicate consistent results among each other will be dangerous. We need carefully designed prospective randomized studies to evaluate any impact of radiation to SVZ in glioblastoma.

Keywords Glioblastoma - Radiotherapy - Radiation · Subventricular zone $\cdot$ Survival $\cdot$ Dose

\section{Introduction}

Despite modern treatment modalities, outcome of glioblastoma is still poor. Median survival is around 14 months $[1,2]$. Standard primary treatment of glioblastoma is surgical resection followed by chemo-radiotherapy and adjuvant chemotherapy with six cycles of Temozolamide [3]. Local recurrence is the main problem during the course of the disease [4].

Neural stem cells are located in the subventricular zone (SVZ) [5]. A growing body of evidence indicates cells with cancer stem cell (CSC) properties causing glioblastoma in preclinical models [6-9]. CSCs are known to be resistant to radiation and chemotherapy [10-12]. Conversely, there are also publications demonstrating that cells residing in SVZ have no proliferative or triggering effect on tumor. Moreover, there is evidence of antitumoral immune-reactive and inflammatory response orginated from the neural stem cells in the SVZ $[13,14]$ against malignancy.

Lim et al. [15] and Adeberg et al. [16] separated glioblastoma cases into categories based on relationship of tumor location to SVZ and/or cortex, reporting that CSCs residing in SVZ may be responsible of glioblastoma 
formation in some cases. Calabrese et al. [17] showed that cells having CSC properties can be ectopically located in any region of the brain where vascular endothelia is present regardless of SVZ. An elegant review describes the advancements of CSC research and the lack of consensus about 'Glioblastome CSCs' [18].

There are four retrospective studies which reported better outcomes with higher ipsilateral SVZ (iSVZ) radiation doses for either overall survival (OS), progression free survival (PFS) or both [19-22], but without any dose consensus. We could only find one abstract with negative results showing no association between iSVZ dose and survival [23]. We doubted whether this situation is related to a publication bias against negative results and whether we can obtain similar results showing any statistical correlation or not, hence the latter becoming the aim of this study.

\section{Materials and methods}

Sixty-six patients with newly diagnosed glioblastoma were treated in our clinic between January 2010 and June 2012. Patients had to be over 18 years old with histopathologically confirmed glioblastoma having at least 1 month of follow-up after the end of concomitant chemo-radiotherapy. Patients who were not treated with standard chemoradiotherapy [3] were excluded. Discontinuation of radiotherapy or chemotherapy during the concomitant phase (2 cases) was also an exclusion criterion. In order to have a minimal follow-up of one year, patients whose treatments were ended within the last year before the data analysis were not included in the study. Pre- and post-operative Magnetic Resonance Imaging (MRI) was mandatory. Patients with multiple tumors, bilaterally extending tumors ( 2 cases), tumors arising from the midline ( 1 case) or from an infratentorial location ( 1 case) were excluded. The remaining 60 patients were included in the study.

Treatment planning system used before 2010 was unavailable. Therefore the backed-up data of 3D conformal radiotherapy plans (3DCRT) before 2010 could not be acquired. Planning tomographies were scanned with $2.5 \mathrm{~mm}$ slice thickness. CTV was defined as resection cavity and residual tumor (if any) identified on gadolinium enhanced $\mathrm{T} 1$ sequence plus a margin of $2 \mathrm{~cm}$ including edema visualized in T2 sequence of MRIs, but at the same time excluding anatomical barriers. PTV margin was $3 \mathrm{~mm}$ around CTV. 60 Gy in 2 Gy daily fractions was prescribed to PTV in single phased 3DCRT plans. Treatments of two patients were planned in Varian Eclipse (Varian Medical Systems, Palo Alto, CA, USA) and the rest in Precise Plan (version 2.16 - Elekta Oncology Systems, Crawley, UK) software, both configured for 6-MV photons.
SVZ volume delineation was done by a single physician according to guidelines [19]. Structures of 3-5 mm thick strips were created lateral to the lateral ventricles. Ipsilateral SVZ were defined as the SVZ on the tumor side. For each patient, contralateral and bilateral SVZ were also created (cSVZ and bSVZ respectively). Dose to each SVZ volume was calculated and measured with dose volume histograms generated on the original plans retrospectively.

All patients were evaluated three monthly after the end of the adjuvant chemotherapy. Progression was defined as radiological verification of recurrence or progression. Additional MRIs were requested in case of clinical suspicion. Patients in undistinguished progression/pseudoprogression status were continued to be followed and confirmed progressions were retrospectively registered with their initial date of suspicion as the date of progression. Decision for salvage treatment depended on mutual discretion of the treating physician, patient and the family.

Information about patients lost to follow-up was acquired from the national database. Descriptive statistics were reported. Date of surgery or biopsy $(\mathrm{Bx})$ was chosen for PFS and OS calculation. Kaplan-Meier method with $95 \%$ confidence intervals (CI) was used for median PFS and OS calculation. Patients lost to follow-up or those who were alive without progression were censored at the time of last follow-up for PFS. Patients who were alive were censored at the time of analysis for OS. CTV, iSVZ, cSVZ and bSVZ doses and CTV volumes were dichotomized each for 25th, 50th (median) and 75th percentiles. Logrank tests for PFS and OS were used for SVZ infiltration $(+/-)$, median age $(\geq 54 ;+/-)$, Karnofsky Performance Status (KPS $\geq 90 ;+/-$ ), gender $(\mathrm{m} / \mathrm{f})$, gross total resection status (GTR; $+/-$ ), doses to CTV, CTV volumes and doses to SVZ structures. Univariate and multivariate Cox proportional hazard models were used to determine the prognostic factors and investigate any relationship between the parameters and survival. Spearman correlation coefficient was used to study the relationship between CTV volume and dose to SVZ's.

All tests were done using JMP 9.0 (SAS Institute Inc. North Carolina, USA). Two-sided $p$ values under 0.05 were considered as statistically significant.

\section{Results}

Descriptive patient characteristics are available on Table 1 . All patients had newly diagnosed glioblastomas. Median follow-up for surviving patients were 24.5 months. Median PFS and OS of the whole cohort were 9.5 (95\% CI 7.7-11.1) and 19.27 (95\% CI 12.77-25.23) months respectively. All patients completed concomitant chemoradiation course without any interruption. 
Table 1 Description of patient and treatment characteristics

\begin{tabular}{|c|c|}
\hline Characteristic & All $(n=60)$ \\
\hline \multicolumn{2}{|l|}{ Age at diagnosis (years) } \\
\hline Mean & $53.1 \pm 12.7$ \\
\hline Median & 54 (range 24-86) \\
\hline \multicolumn{2}{|l|}{ Sex } \\
\hline Female & $21(35 \%)$ \\
\hline Male & $39(65 \%)$ \\
\hline \multicolumn{2}{|l|}{ Extent of resection } \\
\hline GTR & $26(43.3 \%)$ \\
\hline $\mathrm{STR} / \mathrm{Bx}$ & $34(56.7 \%)$ \\
\hline \multicolumn{2}{|l|}{ KPS } \\
\hline$\geq 90$ & $33(55 \%)$ \\
\hline$<90$ & $27(45 \%)$ \\
\hline \multicolumn{2}{|l|}{ Tumor SVZ contact } \\
\hline+ & $32(53.3 \%)$ \\
\hline- & $28(46.7 \%)$ \\
\hline \multicolumn{2}{|l|}{ Tumor side } \\
\hline Left & $27(45 \%)$ \\
\hline Right & $33(55 \%)$ \\
\hline \multicolumn{2}{|l|}{ Lobe where the tumor resides } \\
\hline Frontal & $12(20 \%)$ \\
\hline Parietal & $5(8.3 \%)$ \\
\hline Temporal & $19(31.7 \%)$ \\
\hline Occipital & $4(6.7 \%)$ \\
\hline Intraventricular & $1(1.6 \%)$ \\
\hline Multilobar extension & $19(31.7 \%)$ \\
\hline \multicolumn{2}{|l|}{ CTV } \\
\hline Mean volume $\left(\mathrm{cm}^{3}\right)$ & $299 \pm 158.5$ \\
\hline Quartile volumes (25th, 50th, 75th) & $174.1,280.9,400.2$ \\
\hline Mean dose (Gy) & $62.7 \pm 0.7$ \\
\hline Quartile doses (25th, 50th, 75th) & $62.1,62.7,63.2$ \\
\hline \multicolumn{2}{|l|}{ iSVZ } \\
\hline Mean volume $\left(\mathrm{cm}^{3}\right)$ & $5.2 \pm 2.4$ \\
\hline Mean dose (Gy) & $58.8 \pm 6.5$ \\
\hline Quartile doses (25th, 50th, 75th) & $58.3,61.4,62.2$ \\
\hline \multicolumn{2}{|l|}{ cSVZ } \\
\hline Mean volume $\left(\mathrm{cm}^{3}\right)$ & $6.4 \pm 2.3$ \\
\hline Mean dose (Gy) & $44.9 \pm 15.9$ \\
\hline Quartile doses (25th, 50th, 75th) & $32.3,50.6,59.2$ \\
\hline \multicolumn{2}{|l|}{ bSVZ } \\
\hline Mean volume $\left(\mathrm{cm}^{3}\right)$ & $11.6 \pm 4.2$ \\
\hline Mean dose (Gy) & $51.9 \pm 10.4$ \\
\hline Quartile doses (25th, 50th, 75th) & $43.8,55,60.4$ \\
\hline
\end{tabular}

Log-rank tests showed a significant relation of cSVZ dose $>59.2$ Gy (75th percentile) with poor median PFS (10.37 [95 \% CI 8.37-13.53] vs 7.1 [95\% CI 3.5-8.97] months, $p=0.009$ ) (Fig. 1). PFS were also worse in women than men (median PFS of 7.68 [95 \% CI 4-9] vs 10.77 [95 \% CI

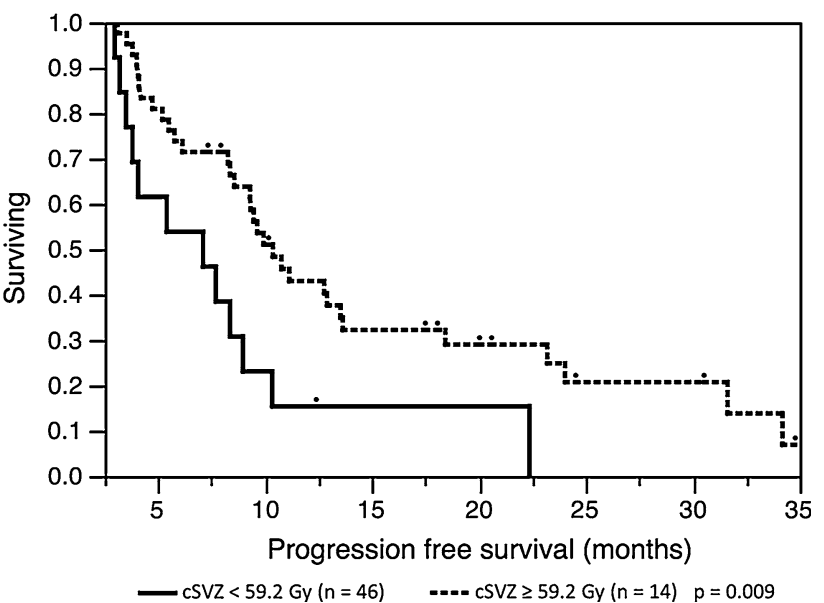

Fig. 1 Progression free survival by contralateral subventricular zone (cSVZ) dose based on log-rank test

8.57-23.2] months, $p=0.009$ ). Those two parameters had significant effect on PFS in univariate Cox proportional hazard analysis, but the effect of high cSVZ dose on PFS lost its statistical significance in multivariate analysis (Table 2) and only sex remained as a significant factor.

Univariate analyses on doses were repeated by stratifying the patients into subgroups of age, sex, resection status, SVZ tumoral contact and KPS (Table 3). cSVZ > 59.2 Gy remained to be a significant prognostic factor for poor PFS in subgroups of age $\geq 54$ (HR: 6.78 [95 \% CI 1.97-24.26], $p=0.003$ ), male gender (HR: 3.96 [95 \% CI 1.36-10.33], $p=0.014$ ), subtotal resection STR/Bx (HR: 8.9 [95 \% CI 2.83-30.9], $p=0.001$ ), SVZ contact positive (HR: 2.56 [95\% CI 1.05-6.19], $p=0.039)$ and KPS $\geq 90$ (HR: 3.43 [95 \% CI 1.09-9.25], $p=0.037$ ). cSVZ dose $>59.2$ Gy was also associated with poor OS in STR/Bx subgroup (HR: 4.83 [95\% CI 1.71-13.97], $p=0.004$ ). High iSVZ dose of $>62.25$ Gy (75th percentile) was associated with poor PFS in both subgroups of high performance status (HR: 2.58 [95 \% CI 1.03-6.05], $p=0.044$ ) (Fig. 2) and SVZ without tumoral contact (HR: $10.57 \quad[95 \%$ CI 2.04-49], $p=0.008$ ) (Fig. 3).

Finally we used Spearman's $\rho$ to identify any correlation between CTV volume and SVZ doses. Although CTV volume was associated with neither PFS nor OS in univariate and multivariate analysis, CTV volume was significantly associated with each SVZ dose ( $\rho$ range $0.39-0.61, p<0.002$ ).

\section{Discussion}

Even today glioblastoma is a devastating disease with poor survival rates and causing gradually deteriorating quality of life. Our patient characteristics and methodology was quite 
Table 2 Treatment outcome analysis of glioblastoma patients with Cox proportional hazards model
${ }^{\text {a }}$ Only parameters with $p<0.1$ included for backward elimination

\begin{tabular}{|c|c|c|c|c|}
\hline \multirow[t]{2}{*}{ Parameter } & \multicolumn{2}{|l|}{ PFS } & \multicolumn{2}{|l|}{ OS } \\
\hline & $\mathrm{HR}(95 \% \mathrm{CI})$ & $p$ value & $\mathrm{HR}(95 \% \mathrm{CI})$ & $p$ value \\
\hline \multicolumn{5}{|l|}{ Univariate analysis } \\
\hline Age $\geq 54$ vs $<54$ & $1.44(0.79-2.66)$ & 0.238 & $1.63(0.87-3.16)$ & 0.131 \\
\hline Sex (f vs m) & $2.83(1.48-5.36)$ & 0.002 & $1.44(0.74-2.71)$ & 0.274 \\
\hline GTR vs STR/Bx & $1.47(0.77-2.80)$ & 0.240 & $1.10(0.55-2.19)$ & 0.785 \\
\hline Tumor contacts SVZ & $1.13(0.62-2.06)$ & 0.700 & $1.24(066-2.37)$ & 0.509 \\
\hline $\mathrm{KPS} \geq 90$ & $1.04(0.58-1.92)$ & 0.888 & $1.17(0.62-2.27)$ & 0.621 \\
\hline \multicolumn{5}{|l|}{ CTV volumes $\left(\mathrm{cm}^{3}\right)$} \\
\hline$>174.1$ & $0.74(0.38-1.53)$ & 0.408 & $0.83(0.41-1.87)$ & 0.638 \\
\hline$>280.9$ & $1.15(0.63-2.09)$ & 0.638 & $1.35(0.72-2.61)$ & 0.352 \\
\hline$>400.2$ & $0.89(0.43-1.72)$ & 0.736 & $0.82(0.38-1.63)$ & 0.580 \\
\hline \multicolumn{5}{|l|}{ CTV doses (Gy) } \\
\hline$>62.1$ & $0.86(0.46-1.73)$ & 0.665 & $0.93(0.47-1.97)$ & 0.845 \\
\hline$>62.7$ & $0.84(0.46-1.53)$ & 0.578 & $0.91(0.48-1.72)$ & 0.769 \\
\hline$>63.2$ & $0.94(0.46-1.79)$ & 0.848 & $0.62(0.28-1.27)$ & 0.197 \\
\hline \multicolumn{5}{|l|}{ iSVZ doses (Gy) } \\
\hline$>58.3$ & $1.43(0.72-3.08$ & 0.318 & $1.12(0.54-2.62)$ & 0.773 \\
\hline$>61.4$ & 1.09 (0.60-1.99) & 0.767 & $0.84(0.44-1.59)$ & 0.591 \\
\hline$>62.2$ & $1.16(0.56-2.24)$ & 0.671 & $1.10(0.52-2.14)$ & 0.800 \\
\hline \multicolumn{5}{|l|}{ cSVZ doses (Gy) } \\
\hline$>32.3$ & $1.04(0.55-2.12)$ & 0.906 & $1.74(0.78-4.61)$ & 0.187 \\
\hline$>50.6$ & $0.98(0.54-1.78)$ & 0.946 & $1.11(0.59-2.13)$ & 0.744 \\
\hline$>59.2$ & $2.42(1.18-4.71)$ & 0.018 & $1.49(0.72-2.88)$ & 0.268 \\
\hline \multicolumn{5}{|l|}{ bSVZ doses (Gy) } \\
\hline$>43.8$ & $0.84(0.43-1.78)$ & 0.640 & $1.18(0.55-2.93)$ & 0.689 \\
\hline$>55$ & $1.02(0.56-1.87)$ & 0.947 & $1.19(0.62-2.30)$ & 0.604 \\
\hline$>60.4$ & $0.97(0.46-1.89)$ & 0.938 & $0.67(0.29-1.37)$ & 0.282 \\
\hline \multicolumn{5}{|c|}{ Multivariate Cox regression ${ }^{a}$} \\
\hline $\operatorname{Sex}(f$ vs $m)$ & $2.39(1.19-4.73)$ & 0.015 & - & - \\
\hline $\mathrm{cSVZ}>59.2$ Gy & $1.72(0.80-3.53)$ & 0.161 & - & - \\
\hline
\end{tabular}

similar with the previous studies [19-22] about this subject: Male/female ratio seems to be around 2:1 [19-22]; the GTR rates [21, 22], performance scores [22] and periventricular tumoral contact ratios [22] are also nearly the same. But most of the dosimetric data and their correlation to outcome do not comply with previously published data. Mean doses to iSVZ, cSVZ and bSVZ are very heterogeneous throughout the published literature. Our dosimetric results are only concordant with those of Gupta et al. [20]. The reason for that might be the difference in techniques or another factor, like tumor location. For example, only Lee et al. [21] did not include patients with multiple and bilateral tumors. Others did not report about this entity or included [20] those patients. Only Chen et al. [22] reported their choice of planning method which was intensity modulated radiotherapy. We do not have information about the technique used by other groups. Total prescribed doses also varied with the greatest range of 30-63 Gy in the study of Evers et al. [19] who also included grade 3 glial tumors. Target volume definition strategy and 60 Gy single phase approach [3] we and Gupta et al. used is widely preferred in European centers whereas two phase approach which is preferred in North America was chosen in the studies of Chen et al. and Lee et al.

The first study including only glioblastoma patients was published as an abstract by Slotman et al. [23] with a cohort of 40 patients. So far it is the only negative study we are aware of. The second study was published by Gupta et al. [20]. They studied 40 glioblastoma cases. Old age $(>50)$, poor recursive partitioning analysis class and increased cSVZ dose were related significantly to worse PFS and OS in univariate survival analysis. In multivariate Cox model, only iSVZ dose was significant for OS. Results being different from the present study may be related to three factors: First, multifocal and bilateral tumors were also included in the study. Second, there is no information 
Table 3 Univariate subgroup analyses with Cox proportional hazard model (only significant results shown)

\begin{tabular}{|c|c|c|c|c|}
\hline \multirow[t]{2}{*}{ Dose stratified under subgroups } & \multicolumn{2}{|l|}{ PFS } & \multicolumn{2}{|l|}{ OS } \\
\hline & $\mathrm{HR}(95 \% \mathrm{CI})$ & $p$ value & $\mathrm{HR}(95 \% \mathrm{CI})$ & $p$ value \\
\hline \multicolumn{5}{|l|}{ Age $\geq 54$} \\
\hline $\mathrm{cSVZ}>59.2 \mathrm{~Gy}$ & $6.78(1.97-24.26)$ & 0.003 & - & - \\
\hline \multicolumn{5}{|l|}{ Male sex } \\
\hline $\mathrm{cSVZ}>59.2 \mathrm{~Gy}$ & $3.96(1.36-10.33)$ & 0.014 & - & - \\
\hline \multicolumn{5}{|l|}{ STR/Bx } \\
\hline $\mathrm{cSVZ}>59.2 \mathrm{~Gy}$ & $8.9(2.83-30.9)$ & 0.001 & $4.83(1.71-13.97)$ & 0.004 \\
\hline \multicolumn{5}{|l|}{ SVZ contact: yes } \\
\hline $\mathrm{cSVZ}>59.2 \mathrm{~Gy}$ & $2.56(1.05-6.19)$ & 0.039 & - & - \\
\hline \multicolumn{5}{|l|}{ SVZ contact: no } \\
\hline iSVZ > 62.2 Gy & $10.57(2.04-49)$ & 0.008 & - & - \\
\hline \multicolumn{5}{|l|}{$\mathrm{KPS} \geq 90$} \\
\hline iSVZ > 62.2 Gy & $2.58(1.03-6.05)$ & 0.044 & - & - \\
\hline $\mathrm{cSVZ}>59.2 \mathrm{~Gy}$ & $3.43(1.09-9.25)$ & 0.037 & - & - \\
\hline
\end{tabular}

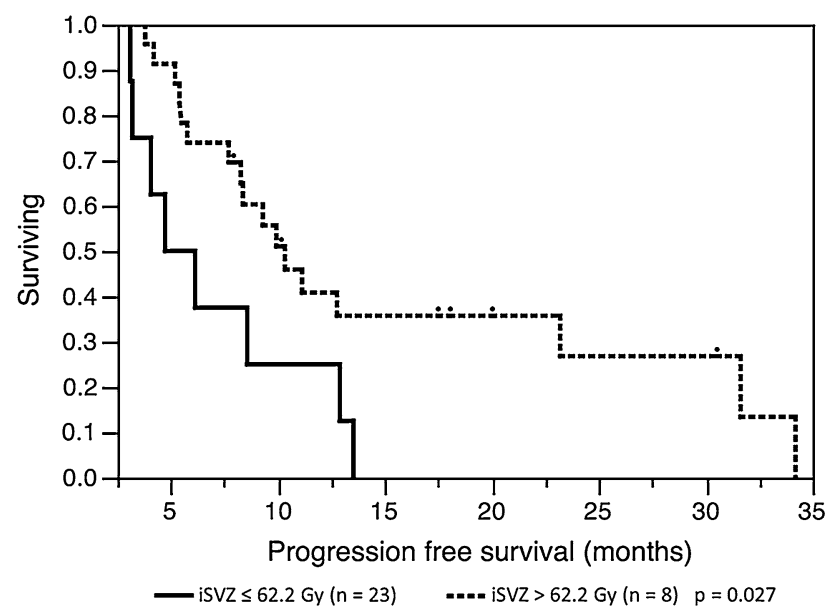

Fig. 2 Progression free survival by ipsilateral subventricular zone (iSVZ) dose in patients having Karnofsky Performance $\geq 90$ based on log-rank test

about associated parameters in the multivariate Cox model being used as nested or not. Third; different from the present study, censored patients had to be with at least 6-months of follow-up after completion of therapy.

The last two studies were published recently. Lee et al. [21] analysed a combined cohort of 173 patients from two centers. This is the largest cohort studied thus far. Twentyone patients received iSVZ dose $>59.4$ Gy and univariate analysis showed a significantly longer median PFS with high dose. Surgery (STR vs GTR and Bx vs GTR), age $>50$ and occipital lobe tumor location were also significantly associated with both PFS and OS in the univariate analysis. In one of the two multivariate Cox models used, iSVZ dose $>59.4$ Gy, surgery (GTR vs STR and GTR vs Bx) and age $<50$ remained to be significantly associated with better

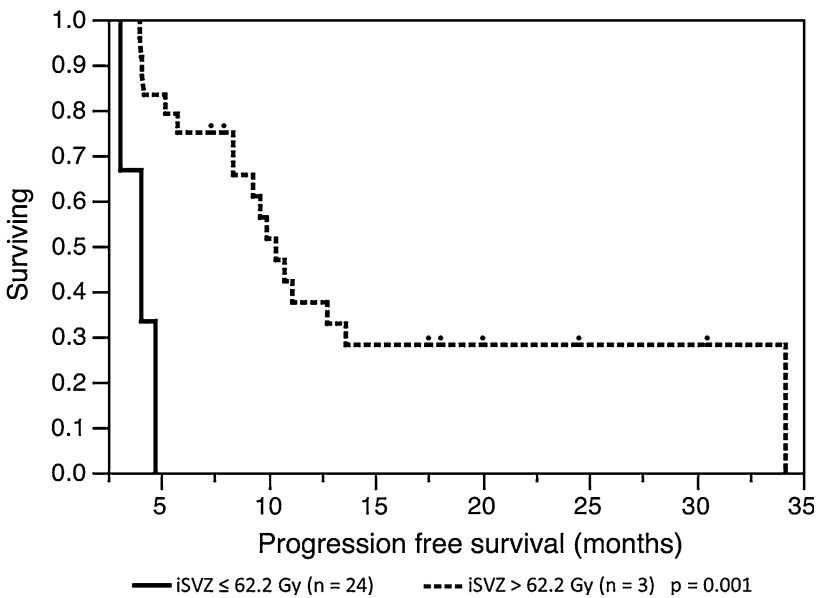

Fig. 3 Progression free survival by ipsilateral subventricular zone dose (iSVZ) in patients having no tumor contact to the subventricular zone based on log-rank test

PFS but only surgery was correlated with better OS. This article has some unique features: This is the only study which also reported the anatomical sites of the tumors. On the other hand, the authors hypothesized at the beginning that 59.4 Gy may be effective to control CSCs, because 59.4-60 Gy is prescribed in glioblastoma as a standard, therefore used this dose threshold in the statistical analysis and the results were supporting the hypothesis. But when they redid the calculation with other arbitrary threshold levels and mean dose ( $>50 \mathrm{~Gy}$ ) to iSVZ, none were associated with significant improvement in PFS. A similar issue is valid for the arbitrary selection of age (50). It is neither mean, median nor some other percentile value. Additionally, our concern for statistics regarding the use of associated values in the same multivariate Cox model in the study of Gupta et al. repeats itself here. 
Chen et al. [22] retrospectively analyzed a set of 116 cases. Minimum follow-up time was 7 months. It is the first and only study reporting the planning technique and the presence of direct tumoral contact to SVZ which was not prognostic. In the univariate analysis, only craniotomy vs biopsy was associated with better PFS. For OS age $<70$ was a better prognostic factor in the subgroups of GTR and STR patients. The subgroup of GTR patients who received $>40$ Gy had significantly improved PFS and OS in the multivariate analysis. But the reason for choosing $40 \mathrm{~Gy}$ as a threshold was not stated in the article.

In our univariate analysis increased cSVZ >59.2 Gy was a strong predictor of PFS and remained to be a significant factor throughout the subgroups of KPS $\geq 90$, age $\geq 54$, male sex, non-GTR and patients with tumoral contact to SVZ. It was a significant factor for worse OS in subgroup without GTR too. Probably the association between increased cSVZ dose and worse PFS is indirectly associated with the extent of disease burden through large CTV volumes. We could not show any significant association between CTV properties and survival but found a significant correlation between CTV volume and SVZ doses. But the same logic should apply to iSVZ dose however mostly it did not: We could only observe a significant impact of increased iSVZ dose $>62.25 \mathrm{~Gy}$ on worse OS in subgroups of KPS $\geq 90$ and patients who did not have any tumor close to ventricles. Finally in the multivariate analysis, only sex remained to be a significant factor on PFS. On the other hand, there is also preclinical evidence about anti-tumoral activity and immune response of stem cells residing in the SVZ against glial malignancies [14]. One can even hypothesize that increased radiation dose destroys stem cells residing in SVZ, which involve in parenchymal repair and generate an immune response against tumor.

Our study has limitations: It has all major drawbacks of a retrospective study with a small sample size. Besides we did not investigate the O6-methylguanine-methyltransferase promoter status which may have changed the results. We did not have any distant recurrences or metachronous primaries and therefore could not analyze its relationship with SVZ parameters if there is any.

Relationship between glioblastoma location, SVZ and dose to CTV and SVZs is a very questionable issue prone to speculative discussion. As we tried to emphasize before, those parameters are somehow connected to each other and therefore susceptible to analytical errors. In a disease with such a poor outcome it is exciting to see data about increasing survival rates just by increased radiation dose. But changing the clinical practice based on retrospective studies which even do not indicate consistent results among each other will be dangerous. However it is definitely a subject worth exploring. We need carefully designed prospective randomized studies to evaluate any impact of radiation to $\mathrm{SVZ}$ in glioblastoma.

Conflict of interest The authors declare that they have no conflict of interest.

Ethical standards The authors declare that the study and reporting methodology complies with the current laws.

\section{References}

1. Koshy M, Villano JL, Dolecek TA et al (2012) Improved survival time trends for glioblastoma using the SEER 17 population-based registries. J Neurooncol 107:207-212

2. Stupp R, Hegi ME, Mason WP et al (2009) Effects of radiotherapy with concomitant and adjuvant temozolomide versus radiotherapy alone on survival in glioblastoma in a randomised phase III study: 5-year analysis of the EORTC-NCIC trial. Lancet Oncol 10:459-466

3. Stupp R, Mason WP, van den Bent MJ et al (2005) Radiotherapy plus concomitant and adjuvant temozolomide for glioblastoma. N Engl J Med 352:987-996

4. Oh J, Sahgal A, Sanghera P et al (2011) Glioblastoma: patterns of recurrence and efficacy of salvage treatments. Can J Neurol Sci 38:621-625

5. Quiñones-Hinojosa A, Sanai N, Soriano-Navarro M et al (2006) Cellular composition and cytoarchitecture of the adult human subventricular zone: a niche of neural stem cells. J Comp Neurol 494:415-434

6. Singh SK, Clarke ID, Terasaki M et al (2003) Identification of a cancer stem cell in human brain tumors. Cancer Res 63:5821-5828

7. Galli R, Binda E, Orfanelli U et al (2004) Isolation and characterization of tumorigenic, stem-like neural precursors from human glioblastoma. Cancer Res 64:7011-7021

8. Bao S, Wu Q, Li Z et al (2008) Targeting cancer stem cells through L1CAM suppresses glioma growth. Cancer Res 68:6043-6048

9. Cheng L, Wu Q, Huang Z et al (2011) L1CAM regulates DNA damage checkpoint response of glioblastoma stem cells through NBS1. EMBO J 30:800-813

10. Bao S, Wu Q, McLendon RE et al (2006) Glioma stem cells promote radioresistance by preferential activation of the DNA damage response. Nature 444:756-760

11. Eramo A, Ricci-Vitiani L, Zeuner A et al (2006) Chemotherapy resistance of glioblastoma stem cells. Cell Death Differ 13: $1238-1241$

12. Firat E, Gaedicke S, Tsurumi C et al (2010) Die Bedeutung der Mitotischen Katastrophe nach $\gamma$-Bestrahlung von Glioblastomstammzellen. Strahlenther Onkol 186(Suppl 1):116

13. Bexell D, Gunnarsson S, Nordquist J, Bengzon J (2007) Characterization of the subventricular zone neurogenic response to rat malignant brain tumors. Neuroscience 147:824-832

14. Mercapide J, Rappa G, Anzanello F, King J, Fodstad O, Lorico A (2010) Primary gene-engineered neural stem/progenitor cells demonstrate tumor-selective migration and antitumor effects in glioma. Int J Cancer 126:1206-1215

15. Lim DA, Cha S, Mayo MC et al (2007) Relationship of glioblastoma multiforme to neural stem cell regions predicts invasive and multifocal tumor phenotype. Neuro Oncol 9:424-429

16. Adeberg S, König L, Bostel T et al (2013) Glioblastomrezidivmuster: radiologische Auswertung des Rezidivmusters von 608 Patienten in Bezug auf neuronale Stammzellen der subventrikulären Zone Adeberg. Strahlenther Onkol 189(Suppl 1):24-25 
17. Calabrese C, Poppleton H, Kocak M et al (2007) A perivascular niche for brain tumor stem cells. Cancer Cell 11:69-82

18. Koshy M, Villano JL, Dolecek TA et al (2012) Improved survival time trends for glioblastoma using the SEER 17 population-based registries. J Neurooncol 107:207-212

19. Evers P, Lee PP, DeMarco J et al (2010) Irradiation of the potential cancer stem cell niches in the adult brain improves progression-free survival of patients with malignant glioma. BMC Cancer 10:384

20. Gupta T, Nair V, Paul SN et al (2012) Can irradiation of potential cancer stem-cell niche in the subventricular zone influence survival in patients with newly diagnosed glioblastoma? J Neurooncol 109:195-203
21. Lee P, Eppinga W, Lagerwaard F et al (2013) Evaluation of high ipsilateral subventricular zone radiation therapy dose in glioblastoma: a pooled analysis. Int J Radiat Oncol Biol Phys 86:609-615

22. Chen L, Guerrero-Cazares H, Ye X et al (2013) Increased subventricular zone radiation dose correlates with survival in glioblastoma patients after gross total resection. Int J Radiat Oncol Biol Phys 86:616-622

23. Slotman BJE, Eppinga WSC, de Haan PF, Lagerwaard J (2011) Is irradiation of potential cancer stem cell niches in the subventricular zones indicated in GBM? (abstr 1058). Int J Radiat Oncol Biol Phys 81(Suppl 1):184 